Ann. Biol. anim. Bioch. Biophys., I971, 11 (2), 225-231.

\title{
DE L'ÉVOLUTION DE QUELQUES ENZYMES SÉRIQUES AU COURS DU GAVAGE DE L'OIE ET DE LEUR CORRELATION AVEC LE POIDS DU FOIE GRAS FINALEMENT OBTENU
}

\author{
B. LECLERCQ et J.-C. BLUM \\ avec la collaboration technique de Liliane Crorzier \\ Station de Recherches avicoles, \\ Centre de Recherche de Tours, I. N. R. A., \\ 37 - Nouzilly \\ RÉSUMḾ́
}

Au cours de trois séries de gavage comportant chacune 30 oies, on a mesuré les variations d'activité de la sérum-glutamique-oxalacétique-transaminase (SGOT), de la sérum-lacticodéshydrogénase (SLDH) et de la sérum-glutamique-pyruvique-transaminase (SGPT). On enregistre toujours une élévation importante de l'activité de ces enzymes sous l'effet du gavage.

Pour la SGPT 1'augmentation est régulière et finalement très forte.

Pour les deux autres enzymes, elle se produit au début du gavage ; l'activité tend ensuite à se stabiliser et dans certains cas à décroître.

Il existe des corrélations positives et significatives entre l'activité de la SGOT et de la SLDH, entre les activités de ces enzymes et le poids du foie prélevé à la fin de l'expérience. Toutefois, le stade de gavage auquel ces corrélations atteignent leur valeur maximum varie d'une série $\lambda$ l'autre. Une corrélation très faible ou nulle relie les valeurs de la SGPT et le poids du foie gras.

\section{INTRODUCTION}

Un grand nombre d'enzymes plasmatiques subissent de profondes modifications lors de certains troubles métaboliques. Les transaminases et la lacticodéshydrogénase sont de ce point de vue les plus étudiées et les plus utilisées en médecine clinique, en particulier pour révéler les cas de dysfonctionnement hépatique, qui au niveau de l'organe se manifestent parfois par une infiltration lipidique. Quelques travaux ont déjà permis d'aborder l'étude de ces phénomènes au cours de la stéatose de l'oie gavée (Tournut et al., I967 ; Szylit et al., I968 ; IVOREC-SzYLII et SzYLIT, r969; Blum, Gaumeton, MUH et LECLERCQ, I970).

Nous présentons ici les résultats de plusieurs essais réalisés grâce à des procédés automatiques d'analyse biochimique et portant sur un grand nombre d'animaux.

Nous essayons au cours de cette étude de relier l'activité enzymatique à un stade donné avec la taille du foie lors de l'abattage. 


\section{MATÉRIEL, E'T MÉTHODES}

Les oies sont de race Landaise. Trois séries de 30 animaux sont gavés successivement à la Station expérimentale d'Artiguères, selon les méthodes utilisées dans cette Station. L'aliment renferme $96,5 \mathrm{p}$. Ioo de maïs, 2,5 p. roo de saindoux et I p. roo de sel. Les animaux en ingèrent quotidiennement l'équivalent de $650 \mathrm{~g}$ sous forme sèche. Au cours des deux premiers essais, des prises de sang sont effectuées avant et pendant le gavage $: 4^{\mathrm{e}}, 8^{\mathrm{e}}$ et $2 \mathrm{I}^{\mathrm{e}}$ jour. Lors du troisième essai, les prélèvements ont lieu le $4^{e}$, le I I $^{\mathrm{e}}$, le $\mathbf{I 4}^{\mathrm{e}}$ et le $2 \mathrm{I}^{\mathrm{e}}$ jour. Les échantillons de sang sont collectés par ponction de la veine alaire. Le sérum est séparé du caillot 24 heures environ après le prélèvement.

$5 \mathrm{ml}$ de chaque sérum sont conservés pour analyse à $-I 5^{\circ} \mathrm{C}$.

Après trois semaines de gavage, les animaux sont sacrifiés et leurs foies pesés.

L'activité de la SGOT est déterminée par la méthode de MorgenSTEN et al. (r966); celle de la SGPT, selon la technique d'AxELson et al. (I965). Enfin, on mesure l'activité de la SLDH grâce à la technique de Morgensten et al. (1965). Toutes ces analyses sont effectuées grâce à l'auto-analyseur Technicon au rythme de 20 échantillons à l'heure.

Une gamme à base de Versatol $E$ (1) permet l'établissement d'une courbe d'étalonnage pour chaque série d'analyse. Pour éviter toute perturbation étrangère à l'effet du gavage, les échantillons hémolysés ne sont pas retenus.

\section{RÉSULTATS}

Dans le tableau I nous rapportons les résultats des deux premières séries de gavage. Le poids du foie reflète l'importance de la stéatose hépatique (BLUM, GRAFF

TABLEAU I

Poids moyen du foie gras (en g), valeur de la SGOT (unités Karmen/ml) de la $S L D H$ (unités Babson/ml) et de la $S G P T$ (unités Henry/ml) au cours des deux premiers essais

\begin{tabular}{|c|c|c|}
\hline & 1er Essai & $2^{e}$ Essai \\
\hline $\begin{array}{l}\text { Nombre d'animaux } \ldots \\
\text { Poids du foie } \ldots \ldots \ldots\end{array}$ & $\begin{array}{r}30 \\
629\end{array}$ & $\begin{array}{r}30 \\
548\end{array}$ \\
\hline 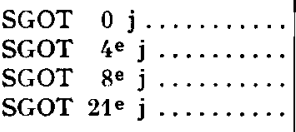 & $\begin{array}{r}29,9 \\
100,2 \\
68,0 \\
74,1\end{array}$ & $\begin{array}{l}30,5 \\
80,4 \\
60,6 \\
78,5\end{array}$ \\
\hline 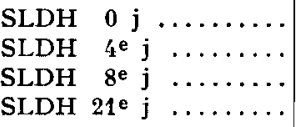 & $\begin{array}{l}439 \\
892 \\
574 \\
738\end{array}$ & $\begin{array}{l}436 \\
750 \\
578 \\
584\end{array}$ \\
\hline 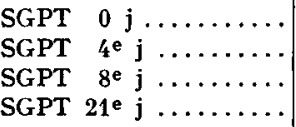 & $\begin{array}{c}0 \\
10,9 \\
77,6 \\
172,5\end{array}$ & $\begin{array}{c}0 \\
18,8 \\
66,0 \\
172,0\end{array}$ \\
\hline
\end{tabular}

(1) Precibio, 2, rue Lhomond, Paris (5e). 
et LECLERCQ, I968); il est en moyenne de $600 \mathrm{~g}$. Il a donc quintuplé du fait de la suralimentation. Après quatre jours de gavage, l'activité de la SGOT est multipliée par un facteur voisin de 3 puis tend à décroître le $8 \mathrm{e}$ jour pour s'élever de nouveau. Une évolution comparable est constatée pour la SLDH bien que 1'ampleur des variations soit plus faible que pour la SGOT. Quant à 1'activité de la SGPT, elle s'élève régulièrement en fonction du temps, passant d'une valeur presque nulle avant le gavage à une valeur voisine de I 72 unités Henry par millilitre en fin de gavage.

TABI EAU 2

Poids moyen du foie gras et valeurs de la SGOT (unités Karmen/ml) de la $S L D H$ (unités Babson/ml) et de la $S G P T$ (unités Henry/ml). au cours $d u 3 \mathrm{e}$ essai

\begin{tabular}{|c|c|}
\hline $\begin{array}{l}\text { Nombre d'animaux } \ldots \ldots \ldots \ldots \ldots \ldots \ldots \ldots \ldots \\
\text { Poids du foie. } \ldots \ldots \ldots \ldots \ldots \ldots \ldots \ldots\end{array}$ & $\begin{array}{r}30 \\
666\end{array}$ \\
\hline 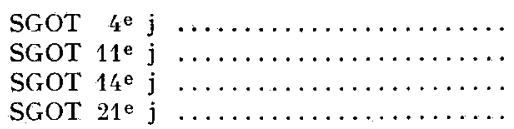 & $\begin{array}{r}54,4 \\
56,4 \\
95,9 \\
155,2\end{array}$ \\
\hline 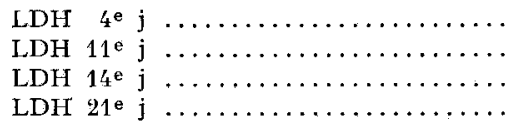 & $\begin{array}{l}889 \\
633 \\
764 \\
813\end{array}$ \\
\hline 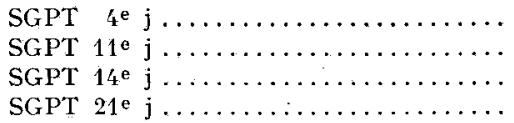 & $\begin{array}{r}74,5 \\
93,4 \\
146 \\
174\end{array}$ \\
\hline
\end{tabular}

Les résultats du troisième essai figurent dans le tableau 2. Les évolutions de la SLDH et de la SGPT sont très semblables à celles observées dans les deux premiers essais. Pour la SGOT, on constate quelques différences; l'activité de cette enzyme s'élevant en fin de gavage.

Nous avons calculé les valeurs des coefficients de corrélation entre l'activité des enzymes étudiées à divers stades et le poids du foie en fin de gavage. L.es résultats relatifs aux deux premiers essais sont rassemblés dans le tableau 3.

Pour la SGOT, la corrélation avec le poids du foie est toujours significative.

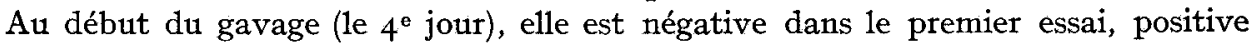
dans le second. Par la suite la corrélation SGOT-poids du foie est toujours positive et particulièrement forte à la fin du gavage. Le $8 \mathrm{e}$ jour, il serait possible de sélectionner les oies aptes à la production du foie gras, mais à ce stade, la corrélation n'est pas très élevée, elle n'augmente guère lorsqu'on remplace la valeur de la SGOT par son accroissement entre o et 8 jours (SGOT 8 e jour — SGOT initial).

La SGPT peut être également reliée au poids du foie de façon positive et significative en fin de gavage; mais cette corrélation demeure faible. Par ailleurs, le rapport $\frac{\text { SGOT }}{\text { SGPT }}$ utilisé classiquement comme inđice du mauvais fonctionnement hépa- 
B. LECLERCQ, J.-C. BLUM

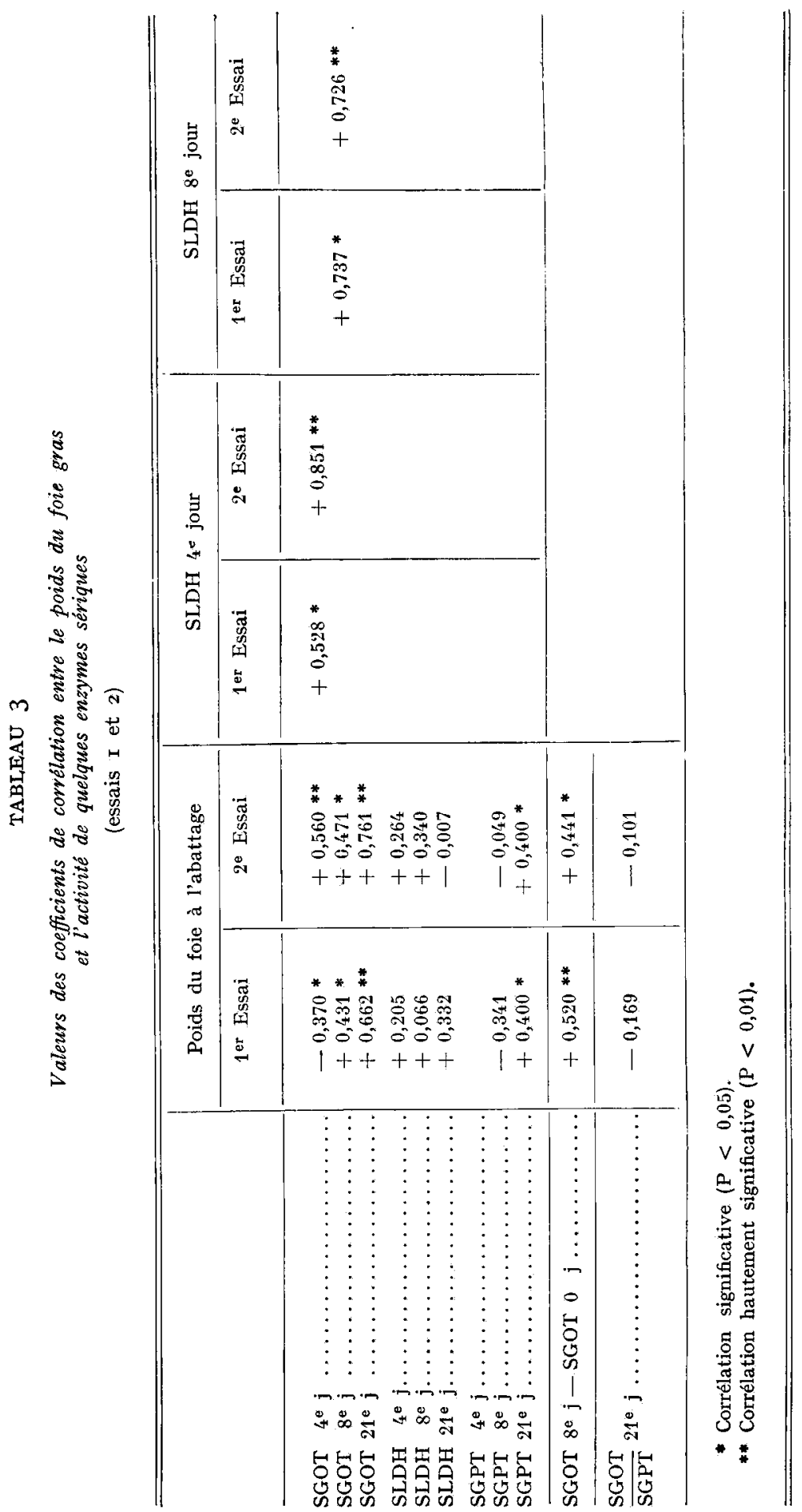


tique n'est ici absolument pas lié au poids du foie. Enfin, on peut observer une corrélation assez étroite entre SLDH et SGOT aussi bien au $4^{\mathrm{e}} \mathrm{qu}$ 'au $8^{\mathrm{e}}$ jour de gavage.

Les valeurs des coefficients de corrélation établies au cours du $3^{\circ}$ essai entre le poids du foie gras à l'abattage et les activités enzymatiques aux $4^{\mathrm{e}}, \mathrm{II}{ }^{\mathrm{e}}, \mathrm{I} 4^{\mathrm{e}}$ et $2 \mathrm{I}^{\mathrm{e}}$ jour de gavage figurent dans le tableau 4.

\section{TABLEAU 4}

Valeurs des coefficients de corrélation entre le poids du foie gras et l'activité de quelques enzymes sériques

(essai 3)

\begin{tabular}{|c|c|}
\hline & Poids du foie à l'abattage \\
\hline $\begin{array}{l}\text { SGOT } 4^{\mathrm{e}} \mathbf{j} \ldots \ldots \ldots \ldots \ldots \ldots \\
\text { SGOT } \mathbf{1 1}^{\mathrm{e}} \mathbf{j} \ldots \ldots \ldots \ldots \ldots \ldots \ldots \\
\text { SGOT } \mathbf{1 4}^{\mathrm{e}} \mathrm{j} \ldots \ldots \ldots \ldots \ldots \ldots \ldots \\
\text { SGOT } 21^{\mathrm{e}} \mathrm{j} \ldots \ldots \ldots \ldots \ldots \ldots\end{array}$ & $\begin{array}{l}+0,214 \\
+0,774 * * \\
+0,435 * \\
+0,200\end{array}$ \\
\hline 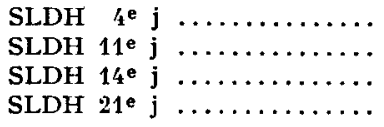 & $\begin{array}{l}+0,365^{*} \\
+0,655 * * \\
+0,627 * * \\
+0,300\end{array}$ \\
\hline 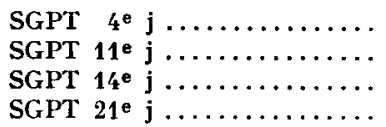 & $\begin{array}{l}-261 \\
-0,019 \\
+0,019 \\
-0,202\end{array}$ \\
\hline
\end{tabular}

* Corrélation significative $(\mathrm{P}<0,05)$.

** Corrélation hautement significative $(\mathrm{P}<0,01)$.

On constate que pour la SGO'T et la SLDH, la corrélation, positive avec le poids $\mathrm{du}$ foie, est significative le $\mathrm{II}^{\mathrm{e}}$ et le $\mathrm{I}_{4}{ }^{\mathrm{e}}$ jour. Sa valeur la plus élevée étant atteinte le II jour, aucune corrélation significative ne peut être mise en évidence avec la SGPT.

\section{DISCUSSION}

La gravité des lésions hépatiques s'accentue au cours du gavage (BLUM, GRAFF

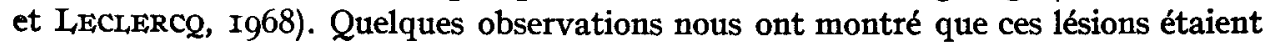
totalement réversibles après 8 jours de gavage, tandis qu'après $2 \mathrm{I}$ jours, elles devenaient irréversibles.

L'activité de la SGPT est nulle chez les animaux non gavés. Son transfert dans le sang signe à coup sûr une atteinte du parenchyme hépatique (OSER, I965). Son augmentation régulière jusqu'à la fin du gavage pourrait rendre compte de la gravité des lésions. Ces lésions ne seraient pas directement liées à l'état de stéatose puisque la corrélation entre le poids du foie et la SGPT est toujours faible.

Etroitement associées et suivant une évolution parallèle, les variations d'activité de la SGOT et de la SLDH ne peuvent pas être interprétées d'une manière simple. 
Pour chaque oie on peut toujours distinguer 3 périodes : augmentation plus ou moins rapide de l'activité dans les premiers jours, phase stationnaire, décroissance ou nouvelle augmentation en fin de gavage.

Nos observations sont donc voisines de celles qu'avaient faites IVOREC-SzYLIT et SzYLIT (1969) à propos de la SLDH. Nous constatons, en outre, que les fluctuations d'activité et la durée des 3 périodes sont plus ou moins grandes selon les individus. Elles dépendent donc de facteurs génétiques et peut-être d'autres facteurs tels que le "stress " au cours du gavage (rôle du gaveur). Dès lors, on peut concevoir que les moyennes enregistrées (tabl. I et 2) puissent être quelque peu différentes selon les troupeaux.

En tout cas on doit remarquer que les activités de la SGOT et de la SLDH peuvent être élevées dès le début du gavage, alors que les lésions hépatiques restant modérées, l'activité métabolique de l'organe augmente considérablement (LECLERCQ et al., I967). On peut admettre que la diffusion de ces enzymes des hépatocytes vers le sang ne résulte pas forcément d'une destruction cellulaire, mais révèle plutôt une modification de la perméabilité des membranes (WELIS, I969). Du fait de la déficience en choline du régime de gavage, la structure des membranes peut être altérée. Cette éventualité est à retenir, car l'infiltration lipidique est particulièrement marquée au début du gavage dans la zone centrolobulaire (BL,UM, GRAF et LECLERCQ I968). Or chez les mammifères la stéatose observée lors de déficience en choline se développe à partir de la zone centrolobulaire, zone particulièrement riche en L,DH (RAPAPORT, I963).

En corrélation avec l'état de stéatose, la libération de GOT et de $\mathrm{LDH}_{\mathrm{D}}$ au début du gavage pourrait être un indice de la déficience en choline, déficience affectant la perméabilité des membranes, mais n'altérant pas réellement l'intégrité du parenchyme hépatique. A la fin du gavage le transfert dans le sang a une signification plus complexe, GOT et $\mathrm{LDH}$ sont des enzymes tissulaires pouvant diffuser comme la GPT après nécrose des cellules.

En définitive, aucune des 3 enzymes sériques dosées au cours du gavage ne permet d'apprécier précocement et à coup sûr le degré de la stéatose hépatique. Il faudrait que la corrélation avec le poids du foie dépasse 0,7 pour qu'il soit possible d'effectuer un tri parmi les animaux. Ce résultat a été obtenu dans le cas de la SGOT le II $^{\mathrm{e}}$ jour de gavage, mais malheureusement au cours d'un setul essai (le $3^{\mathrm{e}}$ ). De nouvelles expériences sont nécessaires pour déterminer les facteurs physiologiques qui peuvent modifier les valeurs de la SGOT. La recherche des isoenzymes et une étude comparée des activités dans le foie et le sérum devraient apporter des éléments d'information supplémentaires.

Reçu pour publication en décembre 1970.

\section{SUMMARY}

CHANGES IN SOME SERUM ENZYMES DURING CRAMMING OF GEESE AND THEIR CORRELATION WITH THE WEIGHT OF FAT IIVER FINALLY OBTAINED

During three series of cramming trials, each with 30 geese, changes in activity of serum glutamic oxaloacetic transaminase (SGOT) serum lactic dehydrogenase (SLDH) and serum 
glutamic pyruvic transaminase (SGPT) were measured. Cramming always caused a large increase in the activity of these enzymes.

For SGPT the increase was regular and finally reached a very high value. For the other two enzymes there was an increase at the beginning of cramming, then values tended to become stable and in some cases to decrease.

There were significant positive correlations between activities of SGOT and SLDH and between the activities of these enzymes and the weight of liver removed at the end of the experiment. However, the stage of cramming at which these correlations were highest differed from one series to another. Between SGPT and weight of fat liver there was no correlation or only a very low one.

\section{RÉFÉRENCES BIBLIOGRAPHIQUES}

Axelson H., Ekman B., Knutson D., 1965. Determination of SGOT, SGPT and alkaline phosphatase with a simplified autoanalyser technique $n$th requiring blank runs. Automation in analytical che. mistry. Mediad Incorporated, New York.

Blum J.-C., GRAfF B., Leclerco B., I968. Study of the biochemical and histochemical process leading to the formation of a fatty liver in the force fed goose. Am. Oil Chem. Soc., 42 nd Fall Meeting. New York.

Blum J..C., Gaumeton M., Muh J., Leclerce B., I970. Modifications des normes sanguines en fonction du degré de stéatose hépatique chez l'oie gavée. Ann. Rech. vét. 1, 167-1 78

Ivorec-Szylit O., Szylit M., r969. Étude des lacticodéshydrogénases plasmatique et hépatique chez l'oie au cours d'une stéatose provoquée par gavage. Ann. Biol. anim. Bioch. Biophys., 9, 205-2I8.

Leclerce B., Durand G., Delpech P., Blum J.-C., ig68. Note préliminaire sur l'évolution des constituants biochimiques du foie au cours du gavage de l'oie. Ann. Biol. anim. Biochim. Biophys., 8, $549-556$.

Morgensten S., Flor R., Kesslerg., KLein B., i965. The automated determination of NAD coupled enzymes. I. Determination of Lactic Deshydrogenase. Automation in Analytical Chemistry. Mediad Incorporated, New York.

Morgensten S., Oklander M., Auerbach J., Kaufman J., Klein B., 1966. Automatic determination of serum glutamic oxalacetic transaminase. Clin. Chem., 12, 95.

Oser B. L., I965. Harek's Physiological chemistry. I. Vol. I4th edition, McGraw-Hill Book Company. New York.

RApaport A. M., 1963. Acinar units and the pathophysiology of the liver. The liver, Roulier édit., Acad. Press, New York, vol. I.

Szylit M., Leclerce B., Ivorec-Szylit O., I968. Relations spécifiques entre les lacticodéshydrogénases đu sérum et du foie chez l'oie : détermination quantitative et caractérisation électrophorétique. C. R. Acad. Sci., Paris, 266, 952-955.

Tournut J., Labie Ch., Espinasse J., Montlaur-Ferradou, 1967. Étude de quelques normes sanguines chez l'oie au cours du gavage. Communication aux Journées de l'oie, Jouy-en-Josas. Résumé in Bull. Soc. Sci. Hyg. Alim. (1969), 57, 137.

WELlS I. C., I969. Release of intracellular enzymes into serum. Can. J. Biochem., 47, 347-352. 